\title{
Fuzzy Sets, Cut Systems and Closure Operators in Sets with Similarities
}

\author{
Jiří Močkoř \\ University of Ostrava, Institute for Research and Applications of Fuzzy Modeling \\ 30.dubna 22, 70103 Ostrava 1, Czech Republic (email: mockor@osu.cz)
}

\begin{abstract}
Closure operators defined on various sets (set of all classical fuzzy sets, set of all semi-cuts, set of all cuts in a Q-set, etc.) are investigated and it is shown how a closure operator defined on one set can be extended to a closure operator defined on another set.
\end{abstract}

Keywords: Residuated lattice, Q-sets, fuzzy sets in Q-sets, cut systems, closure operator

\section{Introduction}

In the fuzzy set theory and fuzzy logic, $\alpha$-cuts of fuzzy sets are used to decompose a fuzzy set into a weighted combination of classical sets using the resolution identity principle. The principle is important in fuzzy set theory because it establishes a bridge between fuzzy sets and crisp sets. Hence, it has been used as the foundation for generalizing concepts and methods based on crisp sets into those based on fuzzy sets (see e.g. [19]). It means that any classical fuzzy set in a set $A$ (i.e. a map from $A$ with values in the unit interval $[0,1])$ can be alternatively expressed as a system of crisp subsets. In fact, recall that a nested system of $\alpha$-cuts in $A$ is a system $\left(C_{\alpha}\right)_{\alpha}$ of subsets of $A$ such that $C_{\alpha} \subseteq C_{\beta}$ if $\alpha \geq \beta$ and the set $\left\{\alpha \in[0,1]: a \in C_{\alpha}\right\}$ has the greatest element for any $a \in A$. Then for any nested system of $\alpha$-cuts $\mathbf{C}=\left(C_{\alpha}\right)_{\alpha}$, a fuzzy set $\mu_{\mathbf{C}}: A \rightarrow[0,1]$ can be constructed such that $\mu_{\mathbf{C}}(x)=\bigvee_{\left\{\beta: x \in C_{\beta}\right\}} \beta$ and, conversely, for any fuzzy set $\mu$ in $A$, a nested system of $\alpha$-cuts is defined by $C_{\alpha}=\{x \in A: \mu(x) \geq \alpha\}$. Between nested systems of $\alpha$-cuts in $A$ and fuzzy sets in $A$ there are some interesting relationships, and from some point of view an investigation of fuzzy sets can be substituted by an investigation of nested systems of $\alpha$-cuts (see e.g. $[1,2])$.

In the fuzzy set theory and its applications some other generalizations of classical fuzzy sets are frequently used. Firstly, instead of the unit interval $[0,1]$ some other structures are used. Most of them are some versions of complete lattices with some additional properties. One of the most frequently used structure is a complete residuated lat-

\footnotetext{
*This work was supported by the European Regional Development Fund in the IT4Innovations Centre of Excellence project (CZ.1.05/1.1.00/02.0070)
}

tice, see e.g. [20] (in some terminology unital and commutative quantale, see [21]), i.e. a structure $Q=(L, \wedge, \vee, \otimes, \rightarrow, 0,1)$ such that $(L, \wedge, \vee)$ is a complete lattice, $(L, \otimes, 1)$ is a commutative monoid with operation $\otimes$ isotone in both arguments and $\rightarrow$ is a binary operation which is residuated with respect to $\otimes$, i.e.

$$
\alpha \otimes \beta \leq \gamma \text { iff } \alpha \leq \beta \rightarrow \gamma
$$

A well known example is the Eukasiewicz algebra $€=\left([0,1], \vee, \wedge, \otimes, \rightarrow_{€}, 0,1\right)$, where

$$
\begin{gathered}
a \otimes b=0 \vee(a+b-1) \\
a \rightarrow_{£} b=1 \wedge(1-a+b) .
\end{gathered}
$$

Further, classical fuzzy sets (or fuzzy sets with values in residuated lattice) are defined in sets. But any set $A$ can be considered as a couple $(A,=)$, where $=$ is a standard equality relation defined in $A$. It is then natural to consider instead of the strict equality relation $=$, some more "fuzzy" equality relation defined in $A$ which is called similarity relation. Hence, instead of a classical set $A$ and a fuzzy set $s: A \rightarrow[0,1]$, we can use a set with similarity relation $(A, \delta)$ (called a $Q$-set) and a "fuzzy set" $s:(A, \delta) \rightarrow Q$.

Our principal goal is to establish some relationships between "fuzzy sets" in $Q$-sets on one side and (nested) systems of $\alpha$-cuts on the other side. Moreover, we will work in a category theory settings, namely we will investigate such relationships not only for sets with similarity relations, but also for morphisms between $Q$-sets in corresponding categories. Categories we will be mostly working with are some analogies of the classical category Set of sets with maps as morphisms.

In our previous papers $[14,16,17]$, we introduced a notion of a fuzzy set in sets with similarity relation $(A, \delta)$ (the so called $Q$-sets), where values of a similarity relation $\delta: A \times A \rightarrow Q$ are from the residuated lattice $Q$. The notion of an $Q$-set was introduced by Fourmann and Scott in [9] for a Heyting algebra $Q$ and we will use it also for a complete residuated lattices. $Q$-sets then represent objects in various categories $\mathbf{K}$ with differently defined morphisms. Among these categories we are interested in two special categories: the category $\operatorname{Set}(Q)$ with morphisms $(A, \delta) \rightarrow(B, \gamma)$ defined as special maps $A \rightarrow B$ and the category $\operatorname{SetR}(Q)$ with morphisms defined as special relations $A \times B \rightarrow Q$. A notion of 
a fuzzy set in $(A, \delta)$ then depends on a category $\mathbf{K}$, i.e. $f$ is a fuzzy set in an $Q$-set $(A, \delta)$ in a category $\mathbf{K}$ (shortly, $f \varlimsup_{\mathbf{K}}(A, \delta)$ ), if $f:(A, \delta) \rightarrow(Q, \leftrightarrow)$ is a morphism in $\mathbf{K}$, where $\leftrightarrow$ is the biresiduation operation in $Q(=$ special similarity relation in $Q)$. This formal extension of classical fuzzy sets enables us to develop the fuzzy set theory in any category of $Q$-sets, with a lot of properties similar to those of classical fuzzy sets. Although such definition of a fuzzy set in a category $\mathbf{K}$ is new, for some concrete examples of a category $\mathbf{K}$ it represents a well known object, in fact. For example, in the category $\operatorname{Set}(Q)$ a fuzzy set in a $Q$-set $(A, \delta)$ is any extensional map $s: A \rightarrow Q$, i.e. a map which is well known and frequently used by various authors (see, e.g., $[5,10,11])$.

In papers $[13,15]$, we proved that fuzzy sets in the categories $\operatorname{Set}(Q)$ and $\operatorname{SetR}(Q)$ can be represented by some cut systems. These results extend in a natural way the above mentioned equivalence between classical fuzzy sets and $\alpha$-cuts. Namely, we proved that any fuzzy set $f \varlimsup_{\operatorname{Set}(Q)}(A, \delta)$ can be represented by the so called f-cut $\mathbf{C}=\left(C_{\alpha}\right)_{\alpha \in Q}$, where $C_{\alpha}$ are subsets of $A$ with some special properties, and, any fuzzy set $g \varlimsup_{\operatorname{SetR}(Q)}(A, \delta)$ can be analogously represented by an f-cut $\mathbf{D}=\left(D_{\alpha}\right)_{\alpha \in Q}$, where $D_{\alpha}$ are subsets of $A \times Q$ also with some special properties. If instead of a similarity relation $\delta$ we will consider standard equality relation $=$, these general theorems represent above mentioned relations between classical fuzzy sets and $\alpha$-cuts.

A relationship between fuzzy sets and f-cuts is even more closer. We show that fuzzy sets structures in sets with similarity relations can be represented by a functor $F_{\mathbf{K}}: \mathbf{K} \rightarrow$ Set from a corresponding category $\mathbf{K}=\operatorname{Set}(Q), \operatorname{SetR}(Q)$ of $Q$-sets into the category of sets and analogously, f-cuts can be represented by a functor $C_{\mathbf{K}}: \mathbf{K} \rightarrow$ Set. A principal relationship between fuzzy sets and f-cuts in $Q$-sets can be then expressed as a natural isomorphism between the functors $F_{\mathbf{K}}$ and $C_{\mathbf{K}}$.

In the paper we want to investigate further properties of fuzzy sets and f-cuts in corresponding categories. As can be expected, not every map $s: A \rightarrow$ $Q$ is a fuzzy set and not every system of subsets $\left(C_{\alpha}\right)_{\alpha}$ of $A$ is an $\mathrm{f}$-cut in a $Q$-set $(A, \delta)$ (in the category $\operatorname{Set}(Q))$. It is then natural to ask the following question: Is it possible to find some completion procedure which extends any such map s or any system $\left(C_{\alpha}\right)_{\alpha}$ onto a fuzzy set or an $f$-cut in the category $\operatorname{Set}(Q)$ or $\operatorname{Set} R(Q)$ ?

In our previous papers $[13,15,16]$ we proved that the answer is positive. Namely, we showed that any system $\mathbf{C}=\left(C_{\alpha}\right)_{\alpha}$ of subsets of $A$ or in $A \times Q$, respectively, can be extended onto an f-cut $\overline{\mathbf{C}}$ in an $Q$-set $(A, \delta)$ in the category $\operatorname{Set}(Q)$ or $\operatorname{SetR}(Q)$, respectively. Moreover, we want to prove that this completion $\mathbf{C} \mapsto \overline{\mathbf{C}}$ is, in some sense, the best possible. To describe the effectiveness of a completion we will use a category theory language. We will use a notion of a reflective subcategory which represents a universal construction how to enhance properties of an object in the most effective way. We will present theorems which state that categories of completions $\overline{\mathbf{C}}$ are reflective subcategories in categories of semicuts $\mathbf{C}$.

Finally we will be interested in closure operators in structures based on sets or $Q$-sets. The notions of a closure system and a closure operator are very useful tools in several areas of classical mathematics. Let us mention classical closure operators in topological spaces, closure operators which enable to extend various mathematical structures to better ones (e.g., metric space to a complete metric space, lattice to a complete lattice, etc.), closure operators in various algebraic structures, e.g., topological groups, etc. All these closure operators have a very similar structure. In fact, if $U$ is an universe for our closure operator (e.g., classical sets, set of all metric spaces, set of all lattices, etc.) with some ordering $\leq$ defined on $U$ (e.g., a set inclusion relation $\subseteq$ ), then a closure operator could be defined as a map $c: U \rightarrow U$ satisfying

$$
\begin{aligned}
& \text { 1. } x \leq c(x) \\
& \text { 2. } c(c(x))=c(x) \\
& \text { 3. } x \leq y \Rightarrow c(x) \leq c(y)
\end{aligned}
$$

for every $x \in U$. This led several authors to investigate the closure operators also in the framework of fuzzy set theory. Recall the papers $[3,4,5,6,7,8]$, where various results about closure operators defined on an universe of classical fuzzy sets are presented. The aim of this paper is to investigate a possibility to extend a closure operator defined on a set of some structures defined on Q-sets to a closure operator defined on another set of such structures.

\section{Preliminary notations and basic results}

To be more self-contained we will introduce several notations which will be used in the paper, and we also recall several results from previous papers which can be useful for full understanding and notation of our results. In the paper, $Q$ denotes a complete residuated lattice $\left(Q, \wedge, \vee, \otimes, \rightarrow, 0_{Q}, 1_{Q}\right)$. Recall that a set with similarity relation (or $Q$-set) is a couple $(A, \delta)$, where $\delta: A \times A \rightarrow Q$ is a map such that

(a) $(\forall x \in A) \quad \delta(x, x)=1$,

(b) $(\forall x, y \in A) \quad \delta(x, y)=\delta(y, x)$,

(c) $(\forall x, y, z \in A) \quad \delta(x, y) \otimes \delta(y, z) \leq \delta(x, z)$ (generalized transitivity).

In the paper, we will use two principal categories with $Q$-sets as objects and with differently defined morphisms. A morphism $f:(A, \delta) \rightarrow(B, \gamma)$ in the first category $\operatorname{Set}(Q)$ is a map $f: A \rightarrow B$ such that $\gamma(f(x), f(y)) \geq \delta(x, y)$ for all $x, y \in A$. The other category $\operatorname{SetR}(Q)$ is an analogy of the category of 
sets with relations between sets as morphisms. Objects of the category $\operatorname{SetR}(Q)$ are the same as in the category $\operatorname{Set}(Q)$ and morphisms $f:(A, \delta) \rightarrow(B, \gamma)$ are maps $f: A \times B \rightarrow Q$ (i.e. $Q$-valued relations) such that

(a) $(\forall x, z \in A)(\forall y \in B) \delta(z, x) \otimes f(x, y) \leq$ $f(z, y)$

(b) $(\forall x \in A)(\forall y, z \in B) \quad f(x, y) \otimes \gamma(y, z) \leq$ $f(x, z)$.

As we mentioned, a fuzzy set $f$ in an $Q$-set $(A, \delta)$ in a category $\mathcal{K}$ (shortly, $f \varlimsup_{\mathcal{K}}(A, \delta)$ ) is a morphism $f:(A, \delta) \rightarrow(Q, \leftrightarrow)$, where $\leftrightarrow$ is the biresiduation operation in $Q$ defined by $\alpha \leftrightarrow \beta=(\alpha \rightarrow$ $\beta) \wedge(\beta \rightarrow \alpha)$. Any classically defined fuzzy set $X$ in a set $A$ with values in $Q$ can be defined equivalently by a system of level sets $X_{\alpha}, \alpha \in Q$, where $X_{\alpha}=\{a \in A: X(a) \geq \alpha\}$. Conversely, any (nested) system $\left(Y_{\alpha}\right)_{\alpha}$ of subsets of $A$ such that for any $a \in A$ the set $\left\{\alpha \in Q: a \in Y_{\alpha}\right\}$ has the greatest element, defines a fuzzy set $Y$ such that $Y(a)=\bigvee_{\left\{\beta: a \in Y_{\beta}\right\}} \beta$. In our previous papers $[13,15]$, we proved that analogously any fuzzy set in $(A, \delta)$ in the category $\operatorname{Set}(Q)$ or in the category $\operatorname{SetR}(Q)$ can be defined equivalently by a system of some special subsets of $A$ or $A \times Q$, respectively, called f-cut, which is defined as follows:

Definition 2.1 Let $(A, \delta)$ be an $Q$-set. Then a system $\mathbf{C}=\left(C_{\alpha}\right)_{\alpha}$ of subsets of $A$ is called an f-cut in $(A, \delta)$ in the category $\operatorname{Set}(Q)$ if

(a) $\forall a, b \in A, \quad a \in C_{\alpha} \Rightarrow b \in C_{\alpha \otimes \delta(a, b)}$,

(b) $\forall a \in A, \forall \alpha \in Q, \quad \bigvee_{\left\{\beta: a \in C_{\beta}\right\}} \beta \geq \alpha \Rightarrow a \in C_{\alpha}$.

Definition 2.2 Let $(A, \delta)$ be an $Q$-set. Then a system $\mathbf{C}=\left(C_{\alpha}\right)_{\alpha}$ of subsets of $A \times Q$ is called an $\mathbf{f}$-cut in $(A, \delta)$ in the category $\operatorname{SetR}(Q)$ if

(a) $C_{\alpha} \subseteq A \times Q$, for any $\alpha \in Q$,

(b) $\forall a, b \in A, \quad(a, \beta) \in C_{\alpha} \Rightarrow(b, \beta) \in C_{\alpha \otimes \delta(a, b)}$,

(c) $\forall a \in A, \forall \gamma \in Q, \quad \bigvee_{\left\{\beta:(a, \gamma) \in C_{\beta}\right\}} \beta \geq \alpha \Rightarrow$ $(a, \gamma) \in C_{\alpha}$

(d) $\forall a \in A, \forall \alpha, \gamma \in Q, \quad(a, \alpha) \in C_{\beta} \Rightarrow(a, \gamma) \in$ $C_{\beta \otimes(\alpha \leftrightarrow \gamma)}$.

In the paper [16], for any $Q$-set $(A, \delta)$ we introduced the following extensional maps, where $F_{\mathbf{K}}(A, \delta)$ is the set of all fuzzy sets in $(A, \delta)$, in the category $\mathbf{K}$.

$$
\begin{gathered}
\widehat{\imath}:\{s: s: A \rightarrow Q \text { is a map }\} \rightarrow F_{\operatorname{Set}(Q)}(A, \delta), \\
\sim:\{s: s: A \times Q \rightarrow Q \text { is a map }\} \rightarrow F_{\operatorname{SetR}(Q)}(A, \delta),
\end{gathered}
$$

defined by the following lemma.

Lemma 2.1 Let $(A, \delta)$ be an $Q$-set and let $s: A \rightarrow$ $Q$ and $g: A \times Q \rightarrow Q$ be maps. Let the maps $\widehat{s}$ and $\widetilde{g}$ be defined by

$$
\begin{gathered}
\widehat{s}(a)=\bigvee_{x \in A} \delta(a, x) \otimes s(x) \\
\widetilde{g}(a, \alpha)=\bigvee_{(x, \beta) \in A \times Q} g(x, \beta) \otimes \delta(x, a) \otimes(\alpha \leftrightarrow \beta),
\end{gathered}
$$

for every $a \in A, \alpha \in Q$. Then $\widehat{s} \in F_{\operatorname{Set}(Q)}(A, \delta)$ and $\widetilde{g} \in F_{\operatorname{SetR}(Q)}(A, \delta)$.

Recall (see $[13,15])$ that also any system of subsets indexed by elements from $Q$ can be extended onto f-cut system. In fact, for any $Q$-set $(A, \delta)$ there exist completion maps

$$
\begin{gathered}
c_{\operatorname{Set}(Q),(A, \delta)}:\left\{\mathbf{C}: C_{\alpha} \subseteq A\right\} \rightarrow C_{\operatorname{Set}(Q)}(A, \delta), \\
c_{\operatorname{SetR}(Q),(A, \delta)}:\left\{\mathbf{D}: D_{\alpha} \subseteq A \times Q\right\} \rightarrow C_{\operatorname{SetR}(Q)}(A, \delta),
\end{gathered}
$$

such that $c_{\operatorname{Set}(Q),(A, \delta)}(\mathbf{C})=\overline{\mathbf{C}}=\left(\overline{C_{\alpha}}\right)_{\alpha}$ and $c_{\operatorname{SetR}(Q),(A, \delta)}(\mathbf{D})=\overline{\mathbf{D}}=\left(\overline{D_{\alpha}}\right)_{\alpha}$, where

$$
\begin{gathered}
\overline{C_{\alpha}}=\left\{a \in A: \bigvee_{\left\{(x, \beta): x \in C_{\beta}\right\}} \beta \otimes \delta(a, x) \geq \alpha\right\}, \\
\text { for any }\left(C_{\alpha}\right)_{\alpha}, C_{\alpha} \subseteq A, \\
\overline{D_{\alpha}}=\left\{(a, \beta) \in A \times Q: \bigvee_{\left\{(x, \tau, \rho):(x, \tau) \in D_{\rho}\right\}} \rho \otimes\right. \\
\delta(a, x) \otimes(\tau \leftrightarrow \beta) \geq \alpha\}
\end{gathered}
$$

for any $\left(D_{\alpha}\right)_{\alpha}, D_{\alpha} \subseteq A \times Q$.

In what follows, we set $Z_{\operatorname{Set}(Q)}(A)=\left\{\mathbf{C}: C_{\alpha} \subseteq\right.$ $A\}$ and $Z_{\operatorname{SetR}(Q)}(A)=\left\{\mathbf{D}: D_{\alpha} \subseteq A \times Q\right\}$.

Example 2.1 Let $\delta$ be the classical identity relation "=" on the set $[0,1]$ and let $Q$ be the Eukasiewicz algebra. Let a system $\mathbf{C}=\left(C_{\alpha}\right)_{\alpha}$ be defined by $C_{\alpha}=[0, \alpha) \subseteq[0,1]$. Then it is clear that $\mathbf{C}$ is not an $f$-cut in $([0,1], \delta)$ in the category $\operatorname{Set}(Q)$. $A$ simple computation shows that $\overline{\mathbf{C}}=([0, \alpha])_{\alpha}$.

In the next parts, for any category $\mathbf{K}=$ $\operatorname{Set}(Q), \operatorname{SetR}(Q)$, we will use the following notations.

$C_{\mathbf{K}}(A, \delta)=\{\mathbf{C}: \mathbf{C}$ is an f-cut in $(A, \delta)$ in $\mathcal{K}\}$.

Principal properties of fuzzy sets and f-cuts in both categories can be described by the following theorems (see $[15,18])$.

Theorem 2.1 Let $f:(A, \delta) \rightarrow(B, \gamma)$ be a morphism in a category $\mathbf{K}=\operatorname{Set}(\mathbf{Q}), \operatorname{Set} R(\mathbf{Q})$.

1. $F_{\text {Set }(Q)}: \operatorname{Set}(Q) \rightarrow$ Set is a functor such that for every $s \in F_{\operatorname{Set}(Q)}(A, \delta), b \in B$, we have $F_{S e t(Q)}(f)(s)(b)=\bigvee_{x \in A} s(x) \otimes \gamma(f(x), b)$.

2. $F_{\operatorname{Set} R(Q)}: \operatorname{Set} R(Q) \rightarrow$ Set is a functor such that for every $t \in F_{\text {SetR }(Q)}(A, \delta), b \in B, \alpha \in Q$, we have $F_{S e t R(Q)}(f)(t)(b, \alpha)=\bigvee_{x \in A} t(x, \alpha) \otimes$ $f(x, b)$.

3. $C_{S e t(Q)}: \operatorname{Set}(Q) \rightarrow$ Set is a functor such that for every $\mathbf{C}=\left(C_{\alpha}\right)_{\alpha} \in C_{S e t(Q)}(A, \delta)$, we have $C_{S e t(Q)}(f)(\mathbf{C})=\left(\overline{f\left(C_{\alpha}\right)}\right)_{\alpha}$. 
4. $C_{\operatorname{SetR}(Q)}: \operatorname{Set} R(Q) \rightarrow$ Set is a functor such that for every $\mathbf{C}=\left(C_{\alpha}\right)_{\alpha} \in C_{S e t R(Q)}(A, \delta)$, we have $C_{\operatorname{Set} R(Q)}(f)(\mathbf{C})=\left(D_{\alpha}\right)_{\alpha}$, where $D_{\alpha}=$ $\left\{(b, \beta) \in B \times Q: \bigvee_{\left\{(a, \tau):(a, \beta) \in C_{\tau}\right\}} f(a, b) \otimes \tau \geq\right.$ $\alpha\}$.

Theorem 2.2 For every category $\mathbf{K}=$ $\operatorname{Set}(Q), \operatorname{SetR}(Q)$ there exists a natural isomorphism

$$
F_{\mathbf{K}} \rightarrow C_{\mathbf{K}}
$$

\section{Properties of completion maps}

As we mentioned in previous Section, for every Qset $(A, \delta)$, there exist completion maps $c_{\mathbf{K},(A, \delta)}$ : $Z_{\mathbf{K}}(A) \rightarrow C_{\mathbf{K}}(A, \delta)$. Elements in $Z_{\mathbf{K}}(A)$ will be called semi-cuts in $(A, \delta)$ in a corresponding category $\mathbf{K}=\operatorname{Set}(Q)$, $\operatorname{SetR}(Q)$, respectively. In this Section we describe some properties of these maps, which show that the completion maps are, in some sense, the best possible. Firstly, by using these completion maps, we can define a lattice structure on the set of all f-cuts $C_{\mathbf{K}}(A, \delta)$, for every category $\mathbf{K}=\operatorname{Set}(Q), \operatorname{SetR}(Q)$.

Definition 3.1 Let $(A, \delta)$ be an $Q$-set.

1. Let $\mathbf{C}_{i}=\left(C_{i, \alpha}\right)_{\alpha}$ be a semi-cut in $(A, \delta)$ in $\mathbf{K}$, for every $i \in I$. Then we set

$$
\bigvee_{i \in I} \mathbf{C}_{i}=\left(\bigcup_{i \in I} C_{i, \alpha}\right)_{\alpha}, \quad \bigwedge_{i \in I} \mathbf{C}_{i}=\left(\bigcap_{i \in I} C_{i, \alpha}\right)_{\alpha} .
$$

2. Let $\mathbf{D}_{i}=\left(D_{i, \alpha}\right)_{\alpha}$ be an f-cut in $(A, \delta)$ in $\mathbf{K}$, for every $i \in I$. Then we set

$$
\bigvee_{i \in I} \mathbf{D}_{i}=\left(\overline{\bigcup_{i \in I} D_{i, \alpha}}\right)_{\alpha}, \quad \bigwedge_{i \in I} \mathbf{D}_{i}=\left(\bigcap_{i \in I} D_{i, \alpha}\right)_{\alpha} .
$$

Proposition 3.1 Let $(A, \delta)$ be a $Q$-set. Then

1. $\left(Z_{\mathbf{K}}(A), \vee, \wedge\right)$ is a complete lattice,

2. $\left(C_{\mathbf{K}}(A, \delta), \vee, \wedge\right)$ is a complete lattice.

Proposition 3.2 Let $(A, \delta)$ be a Q-set. Then the completion map $c_{\mathbf{K},(A, \delta)}: Z_{\mathbf{K}}(A) \rightarrow C_{\mathbf{K}}(A, \delta)$ is a lattice homomorphism.

Using these lattice structures, the completion map can be defined by the following proposition.

Proposition 3.3 Let $(A, \delta)$ be a $Q$-set and let $\mathbf{C} \in$ $Z_{\mathbf{K}}(A, \delta)$. Then

$$
\overline{\mathbf{C}}=\bigwedge\left\{\mathbf{D}: \mathbf{D} \in C_{\mathbf{K}}(A, \delta), \mathbf{C} \subseteq \mathbf{D}\right\}
$$

Analogously as the functor $C_{\mathbf{K}}$, we can define a functor $Z_{\mathbf{K}}: \mathbf{K} \rightarrow$ Set, i.e. for a morphism $f$ : $(A, \delta) \rightarrow(B, \gamma)$, we set

1. for every $\mathbf{C} \in Z_{\operatorname{Set}(Q)}(A), Z_{\operatorname{Set}(Q)}(f)(\mathbf{C})=$ $\left(f\left(C_{\alpha}\right)\right)_{\alpha}$,
2. for every $\mathbf{D} \in Z_{\operatorname{SetR}(Q)}(A), Z_{\operatorname{SetR}(Q)}(f)(\mathbf{D})=$ $\left(f\left(D_{\alpha}\right)\right)_{\alpha}$, where $f\left(D_{\alpha}\right)=\{(b, \beta) \in B \times Q$ : $\left.\bigvee_{\left\{(a, \tau):(a, \beta) \in C_{\tau}\right\}} f(a, b) \otimes \tau \geq \alpha\right\}$.

A principal property of a completion map can be described by the following theorem.

Theorem 3.1 Let $\mathbf{K}$ be any of categories $\operatorname{Set}(Q), \operatorname{Set} R(Q)$ and let $(A, \delta)$ be a $Q$-set. Then the completion map $c_{\mathbf{K},(A, \delta)}$ is a component of a natural transformation $c_{\mathbf{K}}: Z_{\mathbf{K}} \rightarrow C_{\mathbf{K}}$, i.e., for every morphism $f:(A, \delta) \rightarrow(B, \gamma)$, the following diagram commutes.

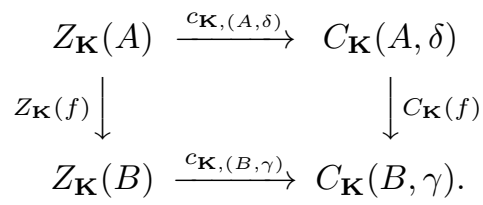

Another principal results of this section are theorems which state that the completion maps are, in some sense, the most effective extensions. To describe those properties of completions we will use a simple category theory language. Namely, we will use the notion of a reflective subcategory which represents a universal construction how to enhance properties of an object in the most effective way (for more details see, e.g., [12]). Recall that a full subcategory $\mathbf{V} \hookrightarrow \mathbf{W}$ of a category $\mathbf{W}$ is a full reflective subcategory in $\mathbf{W}$, if

1. there exists a functor $G: \mathbf{W} \rightarrow \mathbf{V}$,

2. for any object $a$ of $\mathbf{W}$ there exists a morphism $u_{a}: a \rightarrow G(a)$, called $a$ reflection,

3. for any other object $b$ of $\mathbf{V}$ and any other morphism $f: a \rightarrow b$ there exists the unique morphism $\widehat{f}: G(a) \rightarrow b$ such that the diagram commutes:

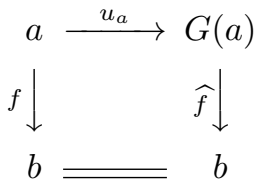

In that case a subcategory $\mathbf{V}$ contains objects with enhanced (or completed) properties (with respect to objects of the category $\mathbf{W}$ ) and from some point of view it represents the most effective extension of objects from the original category W. Many classical examples of well known extensions (or completions) can be expressed as reflective subcategories. Recall a completion of a metric space, a completion of a lattice to a complete lattice, a construction of Abelian groups from noncommutative groups etc. In all these examples full subcategories of objects with these enriched properties are full reflective subcategories in categories of original structures.

We will start with definitions of two categories of cut systems.

Definition 3.2 The objects of the category Cut will be $(A, \delta, \mathbf{C})$, where $(A, \delta)$ is an $Q$-set and $\mathbf{C}$ is an element from $Z_{S e t(Q)}(A)$. Then $f:(A, \delta, \mathbf{C}) \rightarrow$ $(B, \gamma, \mathbf{D})$ is a morphism in Cut if 
(a) $f:(A, \delta) \rightarrow(B, \gamma)$ is a morphism in the category $\operatorname{Set}(Q)$,

(b) $Z_{S e t(Q)}(f)(\mathbf{C}) \subseteq \mathbf{D}$, i.e. $f\left(C_{\alpha}\right) \subseteq D_{\alpha}$ for all $\alpha \in Q$.

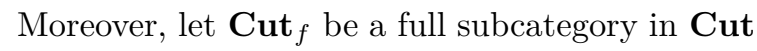
with objects $(A, \delta, \mathbf{C})$ such that $\mathbf{C}$ is an f-cut in $(A, \delta)$ in the category $\operatorname{Set}(Q)$.

Theorem 3.2 The subcategory Cut $\mathbf{C u}_{f} \hookrightarrow$ Cut is a full reflective subcategory in the category Cut, where the reflection is the completion map $c_{S e t(Q),(A, \delta)}$.

An analogical investigation for the category $\operatorname{SetR}(Q)$ is more complicated. We will start with the following definition of $\mathbf{C u t}_{R}$.

Definition 3.3 Objects of $\mathbf{C u t}_{R}$ will be $(A, \delta, \mathbf{C})$, where $(A, \delta)$ is an $Q$-set and $\mathbf{C}$ is a semi-cut, i.e. an element from $Z_{S e t R(Q)}(A)$. Moreover, $f$ : $(A, \delta, \mathbf{C}) \rightarrow(B, \gamma, \mathbf{D})$ is a morphism in $\mathbf{C u t}{ }_{R}$, if

1. $f:(A, \delta) \rightarrow(B, \gamma)$ is a morphism in the category $\operatorname{Set} R(Q)$ and

2. $Z_{S e t R(Q)}(f)(\mathbf{C}) \subseteq \mathbf{D}$, i.e. $f\left(C_{\alpha}\right) \subseteq D_{\alpha}$ for all $\alpha \in Q$, where

$$
\left\{(b, \beta) \in B \times Q: \bigvee_{\left\{(a, \tau):(a, \beta) \in C_{\tau}\right\}}^{f\left(C_{\alpha}\right):=} f(a, b) \otimes \tau \geq \alpha\right\} .
$$

By Cut $_{R, f}$ we denote the full subcategory of the category $\mathbf{C u t}_{R}$ with objects $(A, \delta, \mathbf{C})$, where $\mathbf{C}$ is an object from $C_{\operatorname{SetR}(Q)}(A, \delta)$, i.e. an f-cut.

Theorem 3.3 The subcategory $\mathbf{C u t}_{R, f} \hookrightarrow \mathbf{C u t}_{R}$ is a full reflective subcategory in $\mathbf{C u t}_{R}$, where the reflection is the completion map $c_{S e t R(Q),(A, \delta)}$.

The proof of this theorem requires several technical lemmas, from which the most important is the following lemma describing a continuous property of morphisms in the category $\operatorname{SetR}(Q)$ with respect to the closure operation represented by the completion map.

Lemma 3.1 Let $f:(A, \delta) \rightarrow(B, \gamma)$ be a morphism in the category $\operatorname{Set} R(Q)$ and let $\mathbf{C} \in C_{R}(A)$. Then we have,

$$
f(\overline{\mathbf{C}}) \subseteq \overline{f(\mathbf{C})}
$$

\section{Closure operators}

Closure systems and closure operators play an important role in various mathematical structures, such as topological spaces, lattices, various algebraic structures. In papers $[7,8]$, fuzzy closure operators and systems were studied as natural generalizations of classical closure structures. In this section we continue in developing fuzzy operator theory and we extend the definition of a closure operator to the set of all f-cuts.
Recall firstly some facts about representations of fuzzy sets (in our category $\operatorname{Set}(Q)$ ) by some versions of $\alpha$-cuts. Any classically defined fuzzy set $X$ in a set $A$ with values in $Q$ can be defined equivalently by a system of cuts, defined by

Definition 4.1 Let $A$ be a set. $A$ cut in $A$ is a system $\left(C_{\alpha}\right)_{\alpha \in Q}$ such that

1. $C_{\alpha} \subseteq A$, for every $\alpha \in Q$,

2. if $a \in A$ is such that $\bigvee_{\left\{\beta: a \in C_{\beta}\right\}} \beta \geq \alpha$, then $a \in C_{\alpha}$.

By $D(A)$ we denote the set of all cuts in a set $A$. It is clear that for any $A$, there exists a bijection between the set $F(A)$ of all classical fuzzy sets in a set $A$ and $D(A)$, such that $s \in F(A)$ corresponds to a cut $\left(s_{\alpha}\right)_{\alpha}$, where $s_{\alpha}=\{a \in A: s(a) \geq \alpha\}$ and, conversely, $\left(C_{\alpha}\right)_{\alpha} \in D(A)$ corresponds to a map $s \in F(A)$ such that $s(a)=\bigvee_{\left\{\beta: a \in C_{\beta}\right\}} \beta$.

Let us consider the following universes.

1. The set $2^{A}$ of all subsets of a set $A$,

2. the set $D(A)$ of all cuts in a set $A$,

3. the set $F(A)$ of all classical fuzzy sets in a set $A$, i.e., maps $A \rightarrow Q$,

4. the set $C_{\operatorname{Set}(Q)}(A, \delta)$ of all f-cuts in a Q-set $(A, \delta)$ in the category $\operatorname{Set}(Q)$,

5 . the set $F_{\text {Set }(Q)}(A, \delta)$ of all fuzzy sets in a Q-set $(A, \delta)$ in the category $\operatorname{Set}(Q)$.

We will define a closure operator on the above mentioned universes. Let $A$ be a set, $\delta$ be a similarity relation on $A$ and let $(W, \leq)$ be any of the ordered sets $\left(2^{A}, \subseteq\right),(D(A), \leq),(F(A), \leq$ )$,\left(F_{\operatorname{Set}(Q)}(A, \delta), \leq\right),\left(C_{\operatorname{Set}(Q)}(A, \delta), \leq\right)$, where the order relations $\leq$ are defined point-wise. Then a closure operator on an universe $(W, \leq)$ is a map $c: W \rightarrow W$ such that

1. $x \leq c(x)$, for every $x \in W$,

2. $c(c(x))=x$, for every $x \in W$,

3. $x \leq y \Rightarrow c(x) \leq c(y)$, for every $x, y \in W$.

By $\mathcal{U}(W)$ we denote the set of all closure operators on the universe $W$.

We show several simple examples of closure operators.

Example 4.1 Let $(A, \delta)$ be a Q-set. Let $F_{R}(A)=\{s \mid s: A \times Q \rightarrow Q\}$, $C(A)=\{\mathbf{C} \mid \mathbf{C}$ is a semi-cut in $A\}, C_{R}(A)=$ $\{\mathbf{C} \mid \mathbf{C}$ is a semi-cut in $A \times Q\}$.

1. The extensional map ${ }^{\wedge}: F(A) \rightarrow$ $F_{\text {Set }(Q)}(A, \delta) \subseteq F(A)$ is a closure operator.

2. The extensional map $\sim$ : $F_{R}(A) \rightarrow$ $F_{\operatorname{SetR}(Q)}(A, \delta) \subseteq F_{R}(A)$ is a closure operator.

3. The completion map $C(A) \rightarrow C_{S e t(Q)}(A, \delta) \subseteq$ $C(A)$ is a closure map.

4. The completion map $C_{R}(A) \rightarrow$ $C_{S e t R(Q)}(A, \delta) \subseteq C_{R}(A)$ is a closure map. 
Example 4.2 Let $A$ be a set and let $\delta$ be a similarity relation on $A$. Then by $c_{A, \delta}: 2^{A} \rightarrow 2^{A}$ we denote a map defined by

$$
\forall X \subseteq A, \quad c_{A, \delta}(X)=\left\{a \in A: \bigvee_{x \in X} \delta(a, x)=1\right\}
$$

Then $c_{A, \delta}$ is a closure operator.

The closure operator $c_{A, \delta}$ has some interesting properties. For example, the following lemma holds.

Lemma 4.1 Let $(A, \delta)$ be a $Q$-set and let $s \varlimsup_{S e t(Q)}$ $(A, \delta)$. Then for every $\alpha \in Q, c_{A, \delta}\left(s_{\alpha}\right)=s_{\alpha}$.

Moreover, we can prove some continuity properties of the closure operator $c_{A, \delta}$. Let us consider again a fuzzy set $s \varlimsup_{\operatorname{Set}(Q)}(A, \delta)$. Then $s: A \rightarrow Q$ is a map, and on the set $2^{Q}$ a closure operator $c_{Q, \leftrightarrow}$ can be defined. Then the following lemma holds.

Lemma 4.2 Let $(A, \delta)$ be a $Q$-set and let $s \varlimsup_{\operatorname{Set}(Q)}$ $(A, \delta)$. Then then map

$$
s:\left((A, \delta), c_{A, \delta}\right) \rightarrow\left((Q, \leftrightarrow), c_{Q, \leftrightarrow}\right)
$$

is continuous, i.e., for every $X \subseteq A, s\left(c_{A, \delta}(X)\right) \subseteq$ $c_{Q, \leftrightarrow}(s(X))$.

Another continuity property of the closure operator $c_{A, \delta}$ with respect to a morphisms in the category $\operatorname{Set}(Q)$ is presented in the following lemma.

Lemma 4.3 Let $f:(A, \delta) \rightarrow(B, \gamma)$ be a morphism in the category $\operatorname{Set}(Q)$. Then

$$
f:\left((A, \delta), c_{A, \delta}\right) \rightarrow\left((B, \gamma), c_{B, \gamma}\right)
$$

is a continuous map, i.e., for every $X \subseteq A$, $f\left(c_{A, \delta}(X)\right) \subseteq c_{B, \gamma}(f(X))$ holds.

We want to show an extension principle for closure operators. Namely, we will show how a closure operator defined on a universe can be extended to a closure operator defined on an another universe. We will do it firstly for closure operators defined on the universe constructed from a classical set, i.e., $2^{A}, F(A), D(A)$. We will need here following simple lemma.

Lemma 4.4 Let $A$ be a set and let $\left(C_{\alpha}\right)_{\alpha}$ be a semicut in $A$. We set $\overline{C_{\alpha}}=\left\{a \in A: \bigvee_{\left\{\beta: a \in C_{\beta}\right\}} \beta \geq \alpha\right\}$. Then

(1) $\overline{\mathbf{C}}=\left(\overline{C_{\alpha}}\right)_{\alpha}$ is a cut in $A, \overline{\mathbf{C}} \in D(A)$,

(2) if $\mathbf{C}$ is a cut in $A$, then $\overline{\mathbf{C}}=\mathbf{C}$,

(3) $\overline{\mathbf{C}}$ is the smallest cut such that $\mathbf{C} \subseteq \overline{\mathbf{C}}$.

Lemma 4.5 The map $\mathbf{C} \mapsto \overline{\mathbf{C}}$ is a closure operator defined on the set of all semi-cuts.

Then the following extension theorem holds.
Theorem 4.1 (Extension theorem I) Let $A$ be a set. Then there exist maps such that the diagram commutes.

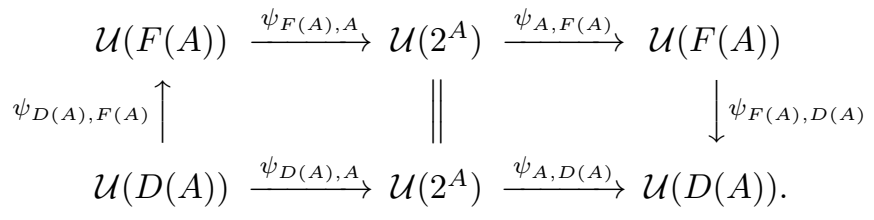

For example, let us show how the map $\psi_{D(A), A}$ is defined. Let $c \in \mathcal{U}(D(A))$ be a closure operator in $D(A)$ and let $X$ be a subset in $A$. Let us consider the constant cut $\mathbf{X}=\left(X_{\alpha}\right)_{\alpha} \in D(A)$, where $X_{\alpha}=$ $X$ for every $\alpha \in Q$. Let us consider the c-closure of $\mathbf{X}$, i.e., $c(\mathbf{X})=\left(C_{\alpha}\right)_{\alpha} \in D(A)$. Then the closure operator $\psi_{D(A), A}(c) \in \mathcal{U}(A)$ is defined by

$\forall X \subseteq A, \quad \psi_{D(A), A}(c)(X)=\left\{a \in A: \bigvee_{\left\{\beta: a \in C_{\beta}\right\}} \beta=1\right\}$

The following theorem then represents an extension theorem for closure systems in sets with similarity relations and corresponding structures, i.e., fuzzy sets and f-cuts.

Theorem 4.2 (Extension theorem II) Let $(A, \delta)$ be an $Q$-set. Then there exist maps such that the following diagram commutes.

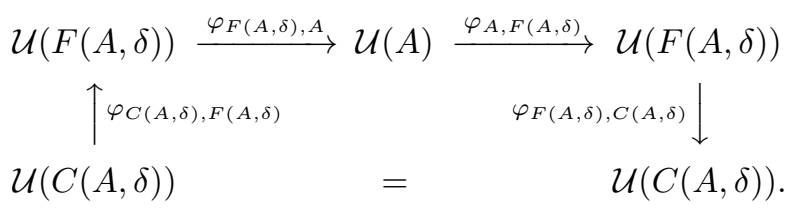

For example, we will show how the map $\varphi_{F(A, \delta), A}$ is defined. Let $c \in \mathcal{U}\left(F_{\operatorname{Set}(Q)}(A, \delta)\right)$ and let $X \subseteq A$. Then the closure operator $\varphi_{F(A, \delta), A}(c)$ is defined by

$$
\varphi_{F(A, \delta), A}(c)(X)=\operatorname{core}(c(\widehat{\chi X})) .
$$

\section{Conclusions}

Properties of completion maps from the set of all semi-cuts to the set of all f-cuts (in a corresponding Q-set $(A, \delta)$ and a category $\mathbf{K})$ are investigated from the point of view of a lattice structure defined on the set of all f-cuts, or from the categorical point of view, as the reflective subcategory construction.

The closure operators are then defined on various sets based on a $\mathrm{Q}$-set $(A, \delta)$, e.g., the set $F(A)$ of all classical fuzzy sets $A \rightarrow Q$, the set $D(A)$ of all cuts in a set $A$, the set $C_{\mathrm{Set}(Q)}(A, \delta)$ of all $\mathrm{f}$-cuts in the category $\operatorname{Set}(Q)$, or the set $F_{\operatorname{Set}(Q)}(A, \delta)$ of all fuzzy sets in $(A, \delta)$ in the category $\operatorname{Set}(Q)$. It was shown how a closure operator defined on one set can be extended to a closure operator defined on another set.

\section{References}

[1] Bělohlávek, R., Fuzzy relational systems, Foundations and Principles, Kluwer Academic Publ., Dordrecht, Boston (2002). 
[2] Bělohlávek, R., Vychodil,V. Fuzzy equational logic, Springer-Verlag, Berlin, Heidelberg (2005).

[3] Bělohlávek, R., Fuzzy closure operators I, J. Math. Anal. Appl. 262(2001), 473-491.

[4] Bělohlávek, R., Fuzzy closure operators II: induced relations, representation and examples, Soft Computing 7(2002), 53-64.

[5] Bělohlávek, R., Fuzzy closure operators induced by similarity. Fundamenta Informaticae 58(2003), 1-13.

[6] Bělohlávek, R., Fuzzy closure operators induced by similarity, Fundamenta Informaticae 58(2003), 1-13.

[7] Biacino, L., Gerla, G., An extension principle for closure operators, J. Math. Anal. Appl. 198(1996), 1-24.

[8] Biacino, L., Gerla, G., Closure systems and $L$-subalgebras, Information Sciences 33(1984), 181-195.

[9] Fourman, M.P., Scott, D.S., Sheaves and logic, Lecture Notes in Mathematics, Vol. 753, Springer Verlag, Berlin, Heidelberg, New York, 1979, 302-401.

[10] Höhle, U.,Fuzzy sets and sheaves. Part I, Basic concepts, Fuzzy Sets and System 158(2007), 1143-1174.

[11] Höhle, U., Fuzzy sets and sheaves Part II: Sheaf-theoretic foundations of fuzzy set theory with applications to algebra and topology, Fuzzy Sets and System 158(2007), 1175-1212.

[12] Mac Lane, S., Categories for the Working Mathematician, Springer-Verlag, Berlin, Heidelberg, New York (1971).

[13] Močkoř, J., Cut systems in sets with similarity relations, Fuzzy Sets and Systems 161(24)(2010), 3127-3140.

[14] Močkoř, J., Fuzzy Sets in Categories of Sets with Similarity Relations, Computational Intelligence, Theory and Applications. Heidelberg, Springer Verlag (2006), 677-682.

[15] Močkoř, J., Fuzzy sets and cut systems in a category of sets with similarity relations, Soft Computing 16(2012), 101-107.

[16] Močkoř, J., Morphisms in categories of sets with similarity relations, Proceedings of IFSA Congress/EUSFLAT Conference. Lisabon (2009), 560-568.

[17] Močkoř, J., Fuzzy objects in categories of sets with similarity relations, Computational Intelligence, Theory and Applications. Heidelberg, Springer (2006), 677-682.

[18] Močkoř, J., alpha-Cuts and models of fuzzy logic, Int. J. Gen. Systems 41(2013), 67-78.

[19] Negoita, C.V., Ralescu, D.A.,Fuzzy Sets and Their Applications, Wiley, New York (1974).

[20] Novák,V., Perfilijeva, I., Močkoř, J., Mathematical principles of fuzzy logic, Kluwer Academic Publishers, Boston, Dordrecht, London (1999).
[21] Rosenthal, K.I., Quantales and Their Applications, Pittman Res. Notes in Math. 234, Longman, Burnt Mill, Harlow, 1990. 